\title{
The combination of CRP isoforms with oxLDL decreases TNF- $\alpha$ and IL-6 release by U937-derived macrophages
}

\author{
IMTISSAL KRAYEM, SAMER BAZZI and MARC KARAM \\ Department of Biology, Faculty of Sciences, University of Balamand, \\ Deir El Balamand, El-Koura, 100-Tripoli, Lebanon
}

Received February 24, 2017; Accepted April 5, 2017

DOI: $10.3892 /$ br.2017.949

\begin{abstract}
C-reactive protein (CRP) and oxidized low density lipoprotein (oxLDL) serve major roles at both early and advanced stages of atherosclerosis. CRP exists in two isoforms, monomeric (m) and pentameric (p), that bring about pro- or anti-inflammatory effects in macrophages. In addition, CRP may form a complex with oxidized low-density lipoprotein (oxLDL) via phosphatidylcholine, thus decreasing its pro-inflammatory effects within macrophages. The aim of the present study was to investigate the single and the combined effects of mCRP, pCRP and oxLDL on U937-derived macrophages. In the current study, U937-derived macrophages were treated in vitro with different combinations of CRP isoforms with or without oxLDL. The levels of major inflammatory cytokines [interleukin (IL)-1 $\beta$, IL-6, IL-8 and tumor necrosis factor (TNF)- $\alpha$ ] along with the production of reactive oxygen species (ROS) were determined. TNF- $\alpha$ and IL-6 levels were significantly decreased $(\mathrm{P}<0.05)$ by the effect of $\mathrm{mCRP}$ and pCRP combined with oxLDL. No significant changes were observed in IL-1 $\beta$, IL-8 or ROS levels.
\end{abstract}

\section{Introduction}

Atherosclerosis is an inflammatory disease of arteries frequently leading to heart attacks and death (1). Investigators have previously suggested that $\mathrm{C}$-reactive protein (CRP) induce atherosclerosis via different signaling pathways (2). Two isoforms of CRP have been identified thus far: pentameric (p)CRP and monomeric (m)CRP (3). Although the role of these isoforms is debatable, the majority of studies suggested anti-inflammatory and pro-inflammatory roles for pCRP and mCRP, respectively (3-6).

Correspondence to: Dr Marc Karam, Department of Biology, Faculty of Sciences, University of Balamand, Deir El Balamand, Kelhat, El-Koura, P.O. Box 100-Tripoli, Lebanon

E-mail: marckaram1@gmail.com

Key words: CRP isoforms, oxLDL, macrophages, inflammation, cytokines
One of the proposed mechanisms underlying CRP action suggests that circulating CRPs initially bind to adherent platelets at the endothelial level of the artery. Subsequently, these activated platelets $(7,8)$ whereby other cells (9) trigger the dissociation of pCRP into five monomers on its membrane. mCRPs activate monocytes through the inflammatory process, as previously described (10).

Both oxLDL and CRP (in its two isoforms) are co-localized in human atherosclerotic plaques and form complexes with glycoproteins (11). In vitro, the complex formed between oxLDL, CRP and lysophosphatidylcholine demonstrates a decreased pro-inflammatory activity suggesting an ability to slow the progression of atherosclerosis (12).

Macrophages in the plaque release different types of cytokines including: interleukin (IL)-1 $\beta$, IL-8 (13), IL-6 (14) and tumor necrosis factor (TNF)- $\alpha$ (15) and produce reactive oxygen species (ROS) (16) to induce inflammation. These molecules affect formation, development and the destabilization of atherosclerotic plaques (17). Moreover, they activate adhesion molecules on endothelial cells causing further recruitment of monocytes in the arteries $(5,10)$. Furthermore, ROS induces vascular diseases by causing endothelial cell dysfunction, increasing inflammatory cell recruitment in the arteries (18).

Previous studies have utilized different models to investigate either the effect of CRP isoforms, or that of pCRP in combination with various forms of LDL, on monocytes and macrophages $(6,19)$. Interestingly, very few studies suggested a possible interaction of these three molecules with plaque cells that may interfere with the plaque development and/or rupture (11). Furthermore, studies investigating CRP effects used CRP preserved with azide, which may cause misleading results with regards to cytokine release (20-23).

In the present study, we investigated the single and combined effects of azide-free CRP isoforms and oxLDL on the release of inflammatory cytokines (IL-1 $\beta$, IL-6, IL-8, TNF- $\alpha$ ) and ROS by U937-derived macrophages in order to obtain a better understanding of the role of these important molecules in the inflammatory process associated with atherosclerosis.

\section{Materials and methods}

CRP monomerization. Human mCRP was obtained by heating human pCRP (catalog. no. 140-11-5; Lee Biosolutions, 
MO, USA) at $80^{\circ} \mathrm{C}$ for $70 \mathrm{~min}$, as previously described (20). CRP monomerization was confirmed by SDS-PAGE $(12.5 \%$ polyacrylamide gel; $20 \mu \mathrm{g}$ protein/lane), which revealed the existence of a $40 \mathrm{kDa}$ band, thus verifying efficient monomerization (data not shown).

U937 cell culture, differentiation and treatment. The human monocytic cell line, U937 (kindly provided by Prof. Marwan El Sabban, American University of Beirut, Beirut, Lebanon), was cultured in growth medium composed of RPMI-1640 medium supplemented with penicillin $(100 \mathrm{U} / \mathrm{ml})$, streptomycin $(100 \mu \mathrm{g} / \mathrm{ml})$, L-glutamine $(2 \mathrm{mM})$ and $10 \%$ fetal bovine serum (FBS; all from Sigma-Aldrich; Merck KGaA, Darmstadt, Germany) and maintained at $37^{\circ} \mathrm{C}$ in a humidified $5 \% \mathrm{CO}_{2}$ atmosphere. Growth medium was replaced every two 2-3 days. U937 monocytes were induced to differentiate into U937-derived macrophages by culturing cells in the presence of $100 \mathrm{nM}$ phorbol-myristate-acetate (PMA; Sigma-Aldrich; Merck KGaA) for $24 \mathrm{~h}$. Macrophages were washed with phosphate buffered saline (PBS) and cultured for an additional $24 \mathrm{~h}$ in growth medium. Following $24 \mathrm{~h}$ culture period, macrophages were washed with PBS, detached via gentle scraping and finally collected by centrifugation at $240 \times \mathrm{g}, 4^{\circ} \mathrm{C}$, for $5 \mathrm{~min}$. Cell viability was evaluated by the trypan blue exclusion method (24) and was $>80 \%$ in all experiments. Macrophages were seeded at an initial density of $7 \times 10^{5}$ viable cells in 24-well plates and allowed to adhere for $24 \mathrm{~h}$. Macrophages were then cultured in growth medium supplemented with polymyxin B (25 $\mu \mathrm{g} / \mathrm{ml}$, Sigma-Aldrich; Merck KGaA) and then either left untreated or treated with $25 \mu \mathrm{g} / \mathrm{ml}$ of mCRP, pCRP, oxLDL (medium oxidized low density lipoprotein, Kalen Biomedical, LLC, Germantown, MD, USA) alone or in combination. Culture supernatants were collected after $24 \mathrm{~h}$ and stored at $-80^{\circ} \mathrm{C}$ for later cytokine analysis.

Enzyme-linked immunosorbent assay. IL-1 $\beta$ (R\&D Systems, Inc., Minneapolis, MN, USA), IL-6, IL- 8 and TNF- $\alpha$ (PeproTech, Inc., Rocky Hill, NJ, USA) levels were assayed in culture supernatants according to the manufacturers' instructions. Samples were analyzed in duplicates and absorbance was measured via Epoch microplate reader (BioTek Instruments, Inc., Winooski, VT, USA).

ROS assay. To evaluate intracellular ROS production, U937-derived macrophages were collected, washed and incubated with $10 \mu \mathrm{M}$ 2',7'-dichlorodihydrofluorescein diacetate $\left(\mathrm{H}_{2} \mathrm{DCF}-\mathrm{DA}\right.$; Thermo Fisher Scientific, Inc., Waltham, MA, USA) for $45 \mathrm{~min}$ at $37^{\circ} \mathrm{C}$ in the dark. Cells were then washed and finally resuspended in cell wash solution (BD Biosciences, Franklin Lakes, NJ, USA). The mean fluorescence intensity of DCF was measured within $30 \mathrm{~min}$ by FACSCalibur and CellQuest software, version 5.1 (BD Biosciences).

Statistical analysis. Statistical analysis was carried out using GraphPad Prism software (version 6, GraphPad Software, Inc., La Jolla, CA, USA) by performing one way analysis of variance followed by Tukey's multiple comparison post hoc test. Data is presented as mean values \pm standard error of the mean. $\mathrm{P}<0.05$ was considered to indicate a statistically significant difference.

\section{Results}

Cytokine release. TNF- $\alpha, \mathrm{IL}-1 \beta, \mathrm{IL}-8$ and IL-6 release by U937-derived macrophages treated with mCRP, pCRP and/or oxLDL for $24 \mathrm{~h}$ was assessed via ELISA. TNF- $\alpha$ levels, produced by U937-derived macrophages, were similar to the control for all samples treated with one of the CRP isoforms alone or in combination with oxLDL. However, the combination of both CRP isoforms significantly reduced TNF- $\alpha$ release by $\sim 1.5$-fold. Similarly, the triple combination demonstrated a similar decrease when compared to the samples treated with pCRP alone or combined with oxLDL (Fig. 1A).

The presence of pCRP and oxLDL in the treated sample lowered the release of IL- 6 by 7-fold when compared to the samples treated with both CRP isoforms or in the combination of mCRP and oxLDL. A further decrease (13-fold) was observed in the triple combination, which was also significant as compared to the samples treated with either isoforms $(\mathrm{P}<0.05$; Fig. 1B).

In contrast, no significant variations were observed neither for IL-1 $\beta$ nor IL- 8 release by U937-derived macrophages under different treatment combinations (Fig. 1C and D).

ROS detection. Although the intracellular ROS level induced by stimulating U937-derived macrophages with $\mathrm{H}_{2} \mathrm{O}_{2}$ (positive control) was significantly high, no significant differences were observed following $24 \mathrm{~h}$ of exposure to different combinations of CRP and oxLDL (P>0.05; Fig. 2).

\section{Discussion}

The role of CRP isoforms has been controversial in the development of atherosclerosis. Although mCRP, pCRP and oxLDL are present at the plaque level, the combined effect of these three factors on the cytokine release by macrophages has not been established yet in vitro. In the present study, we adopted the U937-derived macrophages model to investigate the effect of these factors, as single or in different combinations, on the release of selected inflammatory markers known to be correlated with the atherosclerotic process.

In the current experiment, secreted TNF- $\alpha$ levels were similar in samples treated with one of the parameters added (mCRP, pCRP and oxLDL) and in those containing either one of the CRP isoforms with oxLDL. However, the combination of the isoforms (mCRP with pCRP) with or without oxLDL lowered TNF- $\alpha$ release as compared to untreated cells, cells treated with pCRP alone or with oxLDL.

As expected, IL-6 levels increased in the presence of pCRP or oxLDL; however, this failed to reach statistical significance. Interestingly, IL- 6 released in presence of the double combination lacking mCRP was less than the other treatments, except the one with oxLDL alone. This decrease was more pronounced in the triple combination sample. This lowering of IL- 6 for combinations containing pCRP suggested that pCRP may have the major role in the downregulation of IL-6 release by U937-derived macrophages stimulated with mCRP and oxLDL.

These results suggested a possible interaction between CRP isoforms lowering TNF- $\alpha$ and IL- 6 release by U937-derived macrophages in presence and absence of 
A

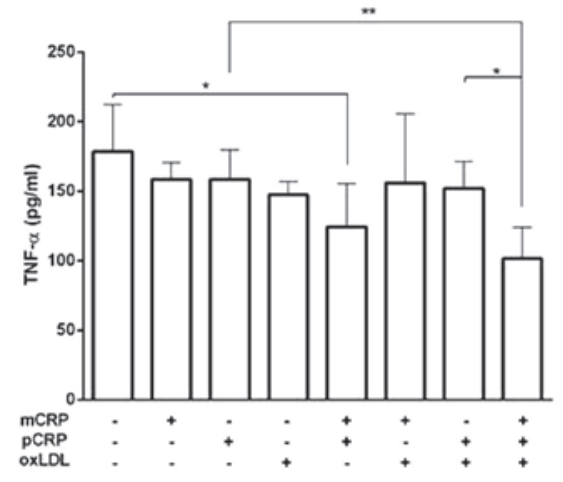

B

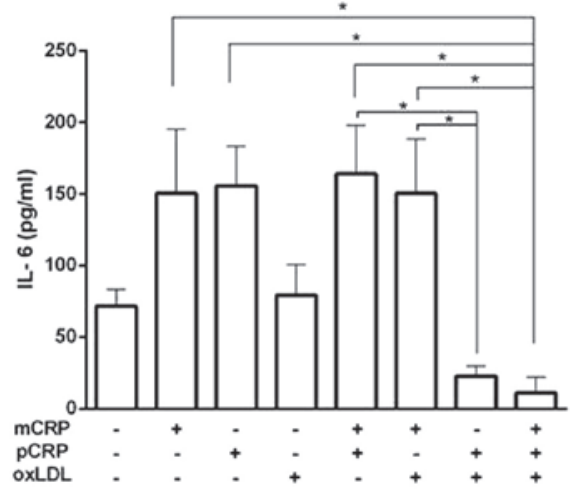

C

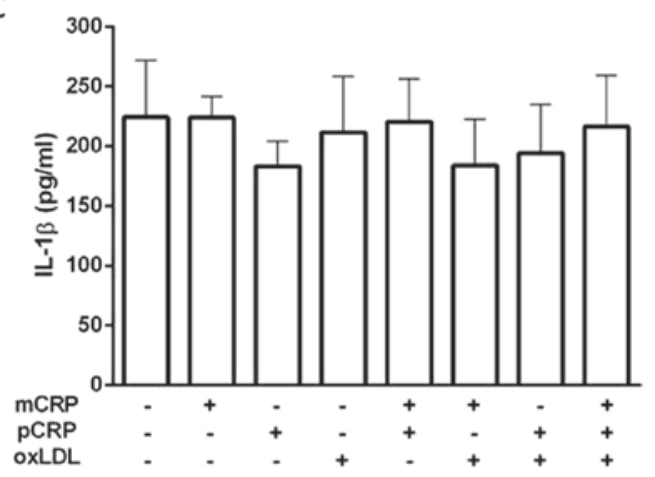

D

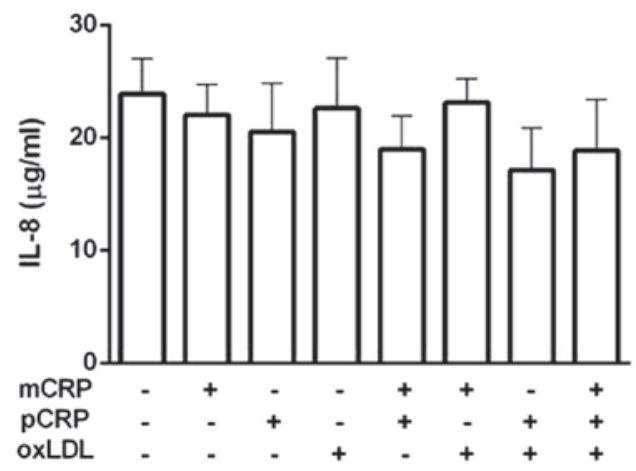

Figure 1. (A) TNF- $\alpha$ (B) IL-6 (C) IL-1 $\beta$ and (D) IL-8 secretion by U937-derived macrophages cultured for $24 \mathrm{~h}$ in the presence (+) or absence (-) of mCRP, pCRP or oxLDL as indicated. Data represent the mean values of cytokine levels \pm standard error of the mean of three independent experiments. ${ }^{*}<0.05$, ${ }^{* *} \mathrm{P}<0.01$ as indicated. TNF- $\alpha$, tumor necrosis factor- $\alpha$; IL, interleukin; mCRP, monomeric C-reactive protein; pCRP, pentameric C-reactive protein; oxLDL, oxidized low-density lipoprotein.

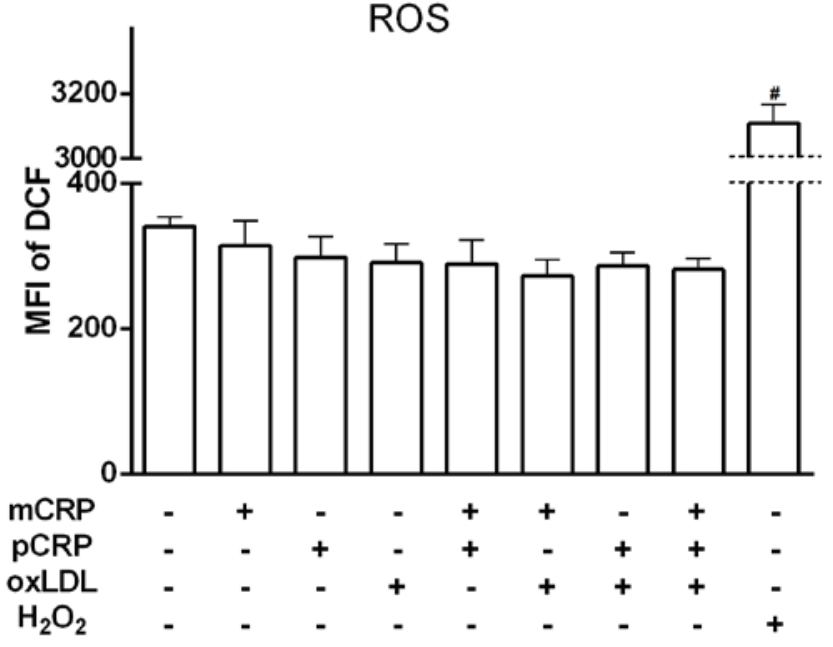

Figure 2. Intracellular ROS production by U937-derived macrophages cultured for $24 \mathrm{~h}$ in the presence $(+)$ or absence $(-)$ of mCRP, pCRP, oxLDL or positive control $\left(\mathrm{H}_{2} \mathrm{O}_{2}\right)$ as indicated. Data indicate the mean values of DCF of MFI \pm standard error of the mean $(n=3) .{ }^{*} \mathrm{P}<0.05$ vs. all other conditions ROS, reactive oxidative species; MFI, mean fluorescence intensity; DCF, dichlorofluorescein; mCRP, monomeric C-reactive protein; pCRP, pentameric C-reactive protein; oxLDL, oxidized low-density lipoprotein.

oxLDL. Therefore, it may be suggested that the presence of mCRP and pCRP decreases TNF- $\alpha$ and IL- 6 secretion by macrophages that may decelerate the process of inflammation.
Previous studies have demonstrated that high level of TNF- $\alpha$ is associated with an increased inflammatory activity in the blood as well as increased levels of IL-6 (25). OxLDL and CRP stimulate activated macrophages to release TNF- $\alpha$, IL-6 and other cytokines that induce vascular and macrophage activation, thus leading to inflammation $(26,27)$. According to the present study, the triple combination reduces the release of these cytokines, which may retard the inflammatory process associated with atherosclerosis. These two pro-inflammatory cytokines levels were increased in elderly and people prone to atherosclerosis (28). Moreover, an increased level of IL-6 exacerbates the atherosclerotic lesions $(19,29)$. Therefore, lowering these pro-inflammatory cytokines release may reduce atherosclerosis progression (30).

Unlike other studies, these results are not influenced by the presence of azide used for CRP preservation that induces TNF- $\alpha$ release $(3,4)$.

The current results support previous studies attributing the pro-inflammatory effects to mCRP rather than pCRP. However, we further propose a possible interaction between the three molecules leading to the observed anti-inflammatory effects on the model cells tested.

Neither IL-1 $\beta$ nor IL- 8 levels were affected by any of the treatment combinations. IL-1 $\beta$, mainly secreted by monocytes and activated macrophages, has an important role in the progression of atherosclerosis (19,31). However, IL-8 serves an important chemotactic role in inflammation and in recruiting neutrophils and other cells to adhere to endothelial cells at the 
site of inflammation. It is also released by various types of cells including key cells involved in atherosclerosis, such as monocytes, macrophages and $\mathrm{T}$ lymphocytes (32).

The levels of released ROS by U937-derived macrophages were similar among all the different treatment conditions. A previous study has reported that CRP induced ROS production by THP-1 macrophages in a time dependent manner (33). However, it was not indicated which CRP isoform was responsible for the increased ROS induction by THP-1 macrophages. Therefore, future experiments will aim to investigate ROS production by U937-derived macrophages following shorter treatment periods with CRP isoforms.

The present study demonstrated that a combination of mCRP and pCRP in the presence of oxLDL would decrease TNF- $\alpha$ and IL-6 production by U937-derived macrophages. However, no significant effects were observed for IL-1 $\beta$ and IL-8, nor for ROS levels in any of the treatments tested. Therefore, the combination of CRP isoforms may stabilize and decelerate the atherosclerotic process. However, additional in vivo and in vitro studies are required to investigate the effects of this combination either on the arteries or on other cell types that have crucial roles in plaque development. Recent studies have been targeting pCRP dissociation as a novel therapy for atherosclerosis (34).

\section{Acknowledgements}

The present study was supported by BIRG (Balamand Internal Research Grant; grant no. 10/2013). The authors would like to thank Dr Zeina Nasr (University of Balamand) and Dr Jad Abdallah (Lebanese American University) as well as Dr Takla El Khoury, Mr. Michel El Zakhem and Mr. Salah El Khatib (University of Balamand) for their assistance and the valuable discussions that enriched this study.

\section{References}

1. Barquera S, Pedroza-Tobías A, Medina C, Hernández-Barrera L, Bibbins-Domingo K, Lozano R and Moran AE: Global overview of the epidemiology of atherosclerotic cardiovascular disease. Arch Med Res 46: 328-338, 2015.

2. Hattori Y, Matsumura M and Kasai K: Vascular smooth muscle cell activation by C-reactive protein. Cardiovasc Res 58: 186-195, 2003.

3. Eisenhardt SU, Thiele JR, Bannasch H, Stark GB and Peter K: C-reactive protein: How conformational changes influence inflammatory properties. Cell Cycle 8: 3885-3892, 2009.

4. Eisenhardt SU, Habersberger J, Murphy A, Chen YC, Woollard KJ, Bassler N, Qian H, von Zur Muhlen C, Hagemeyer CE, Ahrens I, et al: Dissociation of pentameric to monomeric C-reactive protein on activated platelets localizes inflammation to atherosclerotic plaques. Circ Res 105: 128-137, 2009.

5. Khreiss T, József L, Potempa LA and Filep JG: Conformational rearrangement in C-reactive protein is required for proinflammatory actions on human endothelial cells. Circulation 109: 2016-2022, 2004.

6. Schwedler SB, Amann K, Wernicke K, Krebs A, Nauck M, Wanner C, Potempa LA and Galle J: Native C-reactive protein increases whereas modified $\mathrm{C}$-reactive protein reduces atherosclerosis in apolipoprotein E-knockout mice. Circulation 112: 1016-1023, 2005.

7. Eisenhardt SU, Habersberger J and Peter K: Monomeric C-reactive protein generation on activated platelets: The missing link between inflammation and atherothrombotic risk. Trends Cardiovasc Med 19: 232-237, 2009.
8. Filep JG: Platelets affect the structure and function of C-reactive protein. Circ Res 105: 109-111, 2009.

9. Khreiss T, József L, Potempa LA and Filep JG: Loss of pentameric symmetry in C-reactive protein induces interleukin-8 secretion through peroxynitrite signaling in human neutrophils. Circ Res 97: 690-697, 2005.

10. Libby P, Ridker PM and Maseri A: Inflammation and atherosclerosis. Circulation 105: 1135-1143, 2002.

11. Tabuchi M, Inoue $\mathrm{K}$, Usui-Kataoka $\mathrm{H}$, Kobayashi $\mathrm{K}$, Teramoto $\mathrm{M}$, Takasugi K, Shikata K, Yamamura M, Ando K, Nishida K, et al: The association of C-reactive protein with an oxidative metabolite of LDL and its implication in atherosclerosis. J Lipid Res 48: 768-781, 2007.

12. Chang MK, Hartvigsen K, Ryu J, Kim Y and Han KH: The pro-atherogenic effects of macrophages are reduced upon formation of a complex between C-reactive protein and lysophosphatidylcholine. J Inflamm (Lond) 9: 42, 2012.

13. Frostegard J, Ulfgren AK, Nyberg P, Hedin U, Swedenborg J, Andersson $U$ and Hansson GK: Cytokine expression in advanced human atherosclerotic plaques: Dominance of pro-inflammatory (Th1) and macrophage-stimulating cytokines. Atherosclerosis 145: 33-43, 1999.

14. Qiu G, Ho AC, Yu W and Hill JS: Suppression of endothelial or lipoprotein lipase in THP-1 macrophages attenuates proinflammatory cytokine secretion. J Lipid Res 48: 385-394, 2007.

15. Jovinge S, Ares MP, Kallin B and Nilsson J: Human monocytes/macrophages release TNF-alpha in response to Ox-LDL. Arterioscler Thromb Vasc Biol 16: 1573-1579, 1996.

16. Eruslanov E and Kusmartsev S: Identification of ROS using oxidized DCFDA and flow-cytometry. Methods Mol Biol 594: 57-72, 2010.

17. Uzui H, Harpf A, Liu M, Doherty TM, Shukla A, Chai NN, Tripathi PV, Jovinge S, Wilkin DJ, Asotra K, et al: Increased expression of membrane type 3-matrix metalloproteinase in human atherosclerotic plaque: Role of activated macrophages and inflammatory cytokines. Circulation 106: 3024-3030, 2002.

18. Kaneto H, Katakami N, Matsuhisa M and Matsuoka TA: Role of reactive oxygen species in the progression of type 2 diabetes and atherosclerosis. Mediators Inflamm 2010: 453892, 2010.

19. Chávez-Sánchez L, Chávez-Rueda K, Legorreta-Haquet MV, Zenteno E, Ledesma-Soto Y, Montoya-Díaz E, Tesoro-Cruz E, Madrid-Miller A and Blanco-Favela F: The activation of CD14, TLR4, and TLR2 by mmLDL induces IL-1beta, IL-6, and IL-10 secretion in human monocytes and macrophages. Lipids Health Dis 9: 117, 2010.

20. Galve-de Rochemonteix B, Wiktorowicz K, Kushner I and Dayer JM: C-reactive protein increases production of IL-1 alpha, IL-1 beta, and TNF-alpha and expression of mRNA by human alveolar macrophages. J Leukoc Biol 53: 439-445, 1993.

21. Pue CA, Mortensen RF, Marsh CB, Pope HA and Wewers MD: Acute phase levels of C-reactive protein enhance IL-1 beta and IL-1ra production by human blood monocytes but inhibit IL-1 beta and IL-1ra production by alveolar macrophages. J Immunol 156: 1594-1600, 1996.

22. Gershov D, Kim S, Brot N and Elkon KB: C-Reactive protein binds to apoptotic cells, protects the cells from assembly of the terminal complement components, and sustains an antiinflammatory innate immune response: Implications for systemic autoimmunity. J Exp Med 192: 1353-1364, 2000.

23. Taylor KE and van den Berg CW: Structural and functional comparison of native pentameric, denatured monomeric and biotinylated C-reactive protein. Immunology 120: 404-411, 2007.

24. Adan A, Kiraz Y and Baran Y: Cell proliferation and cytotoxicity assays. Curr Pharm Biotechnol 17: 1213-1221, 2016.

25. Bruunsgaard H, Andersen-Ranberg K, Jeune B, Pedersen AN, Skinhoj P and Pedersen BK: A high plasma concentration of TNF- $\alpha$ lpha is associated with dementia in centenarians. J Gerontol A Biol Sci Med Sci 54: M357-M364, 1999.

26. Hansson GK and Hermansson A: The immune system in atherosclerosis. Nat Immunol 12: 204-212, 2011.

27. Devaraj S, Davis B, Simon SI and Jialal I: CRP promotes monocyte-endothelial cell adhesion via Fcgamma receptors in human aortic endothelial cells under static and shear flow conditions. Am J Physiol Heart Circ Physiol 291: H1170-H1176, 2006.

28. Bruunsgaard H, Skinhøj P, Pedersen AN, Schroll M and Pedersen BK: Ageing, tumour necrosis factor-alpha (TNF-alpha) and atherosclerosis. Clin Exp Immunol 121: 255-260, 2000.

29. Huber SA, Sakkinen P, Conze D, Hardin N and Tracy R: Interleukin-6 exacerbates early atherosclerosis in mice. Arterioscler Thromb Vasc Biol 19: 2364-2367, 1999. 
30. Branen L, Hovgaard L, Nitulescu M, Bengtsson E, Nilsson J and Jovinge S: Inhibition of tumor necrosis factor-alpha reduces atherosclerosis in apolipoprotein E knockout mice. Arterioscler Thromb Vasc Biol 24: 2137-2142, 2004.

31. Hansson GK and Libby P: The immune response in atherosclerosis: A double-edged sword. Nat Rev Immunol 6: 508-519, 2006.

32. Harada A, Sekido N, Akahoshi T, Wada T, Mukaida N and Matsushima K: Essential involvement of interleukin-8 (IL-8) in acute inflammation. J Leukoc Biol 56: 559-564, 1994.
33. Zhao XQ, Zhang MW, Wang F, Zhao YX, Li JJ, Wang XP, Bu PL, Yang JM, Liu XL, Zhang MX, et al: CRP enhances soluble LOX-1 release from macrophages by activating TNF- $\alpha$ converting enzyme. J Lipid Res 52: 923-933, 2011.

34. Thiele JR, Zeller J, Bannasch H, Stark GB, Peter K and Eisenhardt SU: Targeting C-reactive protein in inflammatory disease by preventing conformational changes. Mediators Inflamm 2015: 372432, 2015. 